\title{
Acoustooptic spectral estimation: a statistical analysis
}

\author{
Demetri Psaltis and B. V. K. Vijaya Kumar
}

\begin{abstract}
The power spectral density estimate obtained by an acoustooptic (AO) system is analyzed. We find that the variance of the estimate depends on the ratio $T_{2} / T_{1}$, where $T_{1}$ is the aperture of the AO cell and $T_{2}$ is the integration time of the detector.
\end{abstract}

\section{Introduction}

Power spectral density (PSD) estimation is a subject that has been extensively analyzed ${ }^{1,2}$ and is still the subject of intensive research because of its importance in many practical problems. The PSD $W(f)$ of a random process $\{s(x)\}$ is defined as the Fourier transform of the autocorrelation of $\{s(x)\}$ :

$$
W(f)=\int_{-\infty}^{\infty} R(\tau) \exp (-j 2 \pi f \tau) d \tau,
$$

where $R(\tau)$ is the correlation function. One method by which we can obtain an estimate of $W(f)$ is to take the magnitude squared of the Fourier transform of a sample realization of $\{s(x)\}$ :

$$
S(f)=\frac{1}{T_{1}}\left|\int_{-T_{1} / 2}^{T_{1} / 2} s(x) \exp (-j 2 \pi f x) d x\right|^{2}
$$

where $S(f)$ is the PSD estimate. Optical processing systems are very well suited for PSD estimation because the necessary operations in Eq. (2) can be very efficiently realized by a single lens and a square law detector. The data to be processed by an optical computer must modulate an optical beam spatially, and the most efficient way we can achieve this is by using the AO interaction. $^{3}$ Thus there is a great deal of interest in AO spectrum analyzers. ${ }^{4,5}$ A unique feature of these systems is that the Fourier transform is computed over a sliding window of the input data. At different instances in time, the Fourier transform of a different portion of the signal is calculated. Because of the finite integration time of the detector that records the PSD estimate, a large number of these spectra are averaged. In this paper we calculate the expected value and the variance of the PSD estimate obtained by an AO system. (P.

D. Psaltis is with California Institute of Technology, Pasadena, California 91125; B.V.K.V. Kumar is with Carnegie-Mellon University, Department of Electrical Engineering, Pittsburgh, Pennsylvania 15213.

Received 7 July 1980.

0003-6935/81/040601-05\$00.50/0.

(C) 1981 Optical Society of America.
Kellman, ESL Inc., has informed us in a private communication that he and his co-workers have also analyzed this problem independently, and their results are scheduled to appear in Proc. IEEE in January 1981.) The relationship of these statistics to the parameters of the AO system leads to the optimization of the AO PSD estimator.

\section{AO Spectrum Analysis}

A schematic diagram of the AO spectrum analyzer is shown in Fig. 1. The signal $s(t)$ is the sample realization of the random process whose PSD we wish to estimate. $S(t)$ is applied to the AO cell in Fig. 1. The cell is illuminated by a collimated monochromatic light beam incident at the appropriate Bragg angle. The light diffracted by the cell is Fourier transformed by the lens, and the light amplitude at plane $P_{2}$ of Fig. 1 is

$$
F(f, t)=\int_{T_{1}} s(t+x) \exp (-j 2 \pi f x) d x
$$

where $x$ is the spatial coordinate in the direction of propagation of the acoustic wave, $f$ is the spatial frequency, and $T_{1}$ is the aperture of the $\mathrm{AO}$ cell in units of distance. The velocity of sound in the AO material is assumed to be one unit of space/unit of time. A detector array is placed at plane $P_{2}$. The detector responds to the light intensity only, so the light detected at each instant in time is

$$
\begin{aligned}
S(f, t) & =\frac{1}{T_{1}}|F(f, t)|^{2} \\
& =\frac{1}{T_{1}}\left|\int_{-T_{1} / 2}^{T_{1} / 2} s(t+x) \exp (-j 2 \pi f x) d x\right|^{2},
\end{aligned}
$$

where the normalization constant $1 / T_{1}$ has been included in Eq. (4). The light intensity detected is continuously converted to an electronic charge which is accumulated in a potential well (capacitor) adjacent to the detector. The charge is periodically read out and measured. The measured charge is proportional to the total light intensity integrated over the time period that the charge is allowed to accumulate. The output of the detector $P(t)$ is related to the light intensity $S(f, t)$ by 


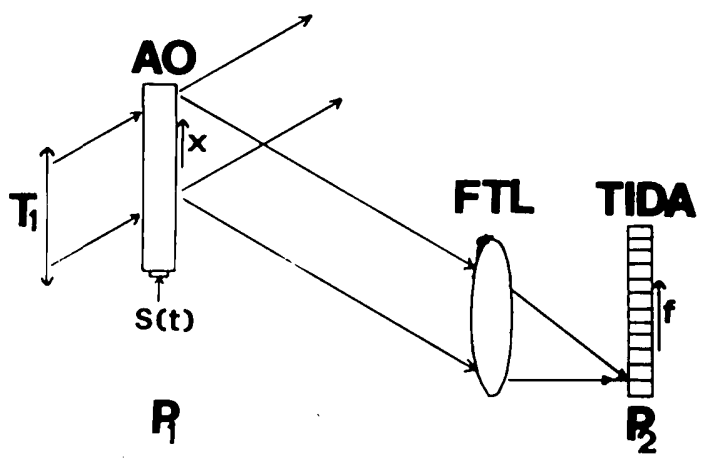

Fig. 1. Acoustooptic spectrum analyzer $(\mathrm{AO}=$ acoustooptic cell, FTL $=$ Fourier transform lens, TIDA $=$ time integrating detector array).

$$
P(f)=\frac{1}{T_{2}} \int_{-T_{2} / 2}^{T_{2} / 2} S(f, t) d t
$$

$T_{2}$ is the integration period of the detector, and $P(f)$ has been normalized by $1 / T_{2}$ as before.

In this paper we are concerned with the consequences of the combination of spatial and temporal integration that the AO processor performs to obtain $P(f)$. In general, the PSD estimate will be contaminated by detector noise and may be otherwise degraded because of imperfections in the $\mathrm{AO}$ processor. We will not take into consideration these effects in most of our discussion.

\section{PSD Estimate Statistics: General Expressions}

To determine how good an estimate of $W(f), P(f)$ is, we must examine its statistics. Specifically we calculate the expected value and the variance of $P(f)$. Given the expected value and the variance we can characterize the estimate by its SNR, where the expected value is the desired signal, and the variance is the energy in the random fluctuations of the estimate about the expected value:

$$
\mathrm{SNR}=\frac{E^{2}[P(f)]}{\operatorname{Var}[P(f)]}
$$

We assume that $s(x)$ is a sample realization from a zero mean, stationary, Gaussian random process.

\section{A. Expected Value of the PSD Estimate}

The expected value of $P(f)$ is

$$
E\{P(f)\}=\frac{1}{T_{2}} \int_{-T_{2} / 2}^{T_{2} / 2} E\{S(f, t)\} d t,
$$

where

$$
\begin{aligned}
E\{S(f, t)\} & =\frac{1}{T_{1}} \iint_{-T_{1} / 2}^{T_{1} / 2} E\{s(x+t) s *(y+t)\} \exp [-j 2 \pi f(x-y)] d x d y \\
& =\frac{1}{T_{1}} \iint_{-T_{1} / 2}^{T_{1} / 2} R_{s}(y-x) \exp [j 2 \pi f(y-x)] d x d y \\
& =\int_{-T_{1}}^{T_{1}}\left(1-\frac{|\tau|}{T_{1}}\right) R_{s}(\tau) \exp (j 2 \pi f \tau) d \tau \\
& =W(f) *\left\{T_{1} \operatorname{sinc}^{2}\left(f T_{1}\right)\right\} .
\end{aligned}
$$

$R_{s}(\tau)$ is the correlation of the random process $\{s(x)\}, \tau$ $=y-x$, and $\operatorname{sinc}(x)=(\sin \pi x) /(\pi x)$. Since $E\{S(f, t)\}$ does not depend on $t$ for a stationary process, as evidenced from Eq. (8), we find from Eq. (7)

$$
E\{P(f)\}=E\{S(f, t)\}=W(f) *\left\{T_{1} \operatorname{sinc}^{2}\left(f T_{1}\right)\right\},
$$

where $*$ denotes convolution.

As we see from Eq. (9), the temporal integration on the detector does not affect the expected value of the PSD estimate. The aperture $T_{1}$, over which the spatial integration is performed, introduces blurring by the $\operatorname{sinc}^{2}$ function, which limits the frequency resolution of the PSD estimate to $1 / T_{1}$. As $T_{1}$ approaches infinity, the function $T_{1} \operatorname{sinc}^{2}\left(f T_{1}\right)$ approaches a $\delta$ function, and $E[P(f)]$ approaches $W(f)$. Thus $P(f)$ is an asympototically unbiased estimator of $W(f)$.

\section{B. Variance of the PSD Estimate}

The variance of $P(f)$ is defined as

$$
\begin{aligned}
\operatorname{Var}\{P(f)\} & =E\left\{P(f) P^{*}(f)\right\}-|E\{P(f)\}|^{2} \\
& =E\left\{\frac{1}{T_{2}^{2}} \iint_{-T_{2} / 2}^{+T_{2} / 2} S(f, t) S^{*}(f, u) d t d u\right\}-|E\{S(f)\}|^{2} \\
& =\frac{1}{T_{2}^{2}} \iint_{-T_{2} / 2}^{+T_{2} / 2}\left[E\left\{S(f, t) S^{*}(f, u)\right\}-|E\{S(f)\}|^{2}\right] d t d u .
\end{aligned}
$$

We use the expression for $S(f, t)$ from Eq. (4) to evaluate the term in the square brackets in the above equation:

$$
\begin{aligned}
& E\left\{S(f, t) S^{*}(f, u)\right\}-|E\{S(f)\}|^{2} \\
& =\frac{1}{T_{1}^{2}} \iiint \int_{-T_{1} / 2}^{+T_{1} / 2} E\{s(x+t) s(y+t) s(p+u) s(q+u)\} \\
& \quad \cdot \exp [-j 2 \pi f(x-y+p-q)] d x d y d p d q-|E\{S(f)\}|^{2} .
\end{aligned}
$$

The fourth-order moment in the above equation can be expanded as the sum of three products of second-order moments (correlations). This is possible because of the Gaussian assumption. ${ }^{6}$ One of the three products is equal to $|E\{S(f)\}|^{2}$, and it is canceled in Eq. (11). We use the remaining two terms in Eq. (11) to obtain the following expression for the variance of $P(f)$ :

$$
\begin{aligned}
\operatorname{Var}\{P(f)\}= & \frac{1}{T_{2}^{2}} \iint_{-T_{2} / 2}^{+T_{2} / 2} d t d u\left[1 / T_{1}^{2} \iiint \int_{-T_{1} / 2}^{+T_{1} / 2}\right. \\
& \times \exp [-j 2 \pi f(x-y+p-q)] \\
& \cdot\left\{R_{s}(p-x+u-t) R_{s}(q-y+u-t)\right. \\
& \left.\left.+R_{s}(q-x+u-t) R_{s}(p-y+u-t)\right\} d x d y d p d q\right]
\end{aligned}
$$

This rather complicated expression does convey a very significant fact: the variance of $P(f)$, and hence the SNR, depends on both the temporal and spatial integration parameters $\left(T_{1}\right.$ and $\left.T_{2}\right)$. To provide a better understanding of how these parameters determine SNR, we calculate the variance of $P(f)$ for the case of white noise in the next section. In the following section we extend our results and conclusions to the case of nonwhite noise. 


\section{PSD Estimate Statistics: White Noise}

For a white noise process, $R_{s}(\tau)=R_{0} \delta(\tau)$. For this case the expected value of $P(f)$ is obtained by substituting into Eq. (9) the above correlation function:

$$
E\{P(f)\}=E\{S(f)\}=R_{0} .
$$

Thus the estimate is unbiased, in this case, for all values of $T_{1}$. Before we proceed with the calculation of the variance of $P(f)$, it is appropriate to recall that the variance of $S(f)$ (the PSD estimate we would obtain with no time integration) is ${ }^{1}$

$$
\operatorname{Var}\{S(f)\}=R_{0}^{2}\left\{1+\operatorname{sinc}^{2}\left(2 f T_{1}\right)\right\} .
$$

The SNR of the instantaneous estimate is

$$
\begin{aligned}
\operatorname{SNR}\{S(f)\} & =\frac{|E\{S(f)\}|^{2}}{\operatorname{Var}\{S(f)\}}=\frac{R_{0}^{2}}{R_{0}^{2}\left\{1+\operatorname{sinc}^{2}\left(2 f T_{1}\right)\right\}} \\
& =\left[1+\operatorname{sinc}^{2}\left(2 f T_{1}\right)\right]^{-1} .
\end{aligned}
$$

If the $S(f)$ is sampled at discrete points along the frequency axis, by placing these samples at $f=n / 2 T_{1}$ (where $n$ is an integer), we obtain the maximum SNR equal to unity. If no temporal integration is performed, we obtain SNR equal to 1 independently of the choice of the parameter $T_{1}$. Thus this would be an inconsistent estimate of $W(f)$. We now return our attention to the calculation of the variance of $P(f)$ for the case of white noise, which we obtain by substituting $R_{s}(\tau)=$ $R_{0} \delta(\tau)$ in Eq. (12):

$\operatorname{Var}\{P(f)\} \simeq \begin{cases}R_{0}^{2}\left[1-2 / 3\left(T_{2} / T_{1}\right)+1 / 6\left(T_{2} / T_{1}\right)^{2}\right] & \text { for } T_{1}>T_{2} \\ R_{0}^{2}\left[2 / 3\left(T_{1} / T_{2}\right)-1 / 6\left(T_{1} / T_{2}\right)^{2}\right] & \text { for } T_{2}>T_{1} .\end{cases}$

The derivation of Eq. (16) is presented in the Appendix. The SNR of $P(f)$ is given by

$\operatorname{SNR}\{P(f)\} \simeq\left\{\begin{array}{l}{\left[1-2 / 3\left(T_{2} / T_{1}\right)+1 / 6\left(T_{2} / T_{1}\right)^{2}\right]^{-1}} \\ {\left[2 / 3\left(T_{1} / T_{2}\right)-1 / 6\left(T_{1} / T_{2}\right)^{2}\right]^{-1}}\end{array}\right.$ for $T_{1}>T_{2}$ for $T_{2}>T_{1}$.

For $T_{2} \gg T_{1}$,

$$
\operatorname{SNR}\{P(f)\} \simeq 3 / 2\left(T_{2} / T_{1}\right),
$$

and of course as $T_{2}$ tends to infinity the variance becomes zero. Thus in this case $P(f)$ is a consistent estimate. As evidenced by Eqs. (17)-(18) the SNR is determined by the ratio of the spatial aperture to the temporal integration period.

In Fig. 2 we plot the SNR as a function of the ratio $T_{2} / T_{1}$ and also as a function of $T_{2}$ for three typical values of $T_{1} . \quad T_{1}=0.5 \mu \mathrm{sec}$ corresponds to a surface wave AO spectrum analyzer, $T_{1}=5 \mu$ sec corresponds to a wide-bank bulk device ( $\mathrm{LiNb})$, and $T_{1}=50 \mu \mathrm{sec}$ to a fine frequency resolution bulk device $\left(\mathrm{TeO}_{2}\right)$.

It is obvious from Eq. (17) and Fig. 2 that for a fixed SNR, the temporal integration required varies drastically for the various implementations of $\mathrm{AO}$ spectrum analyzers. Conversely, for a fixed integration time $T_{2}$, one can actually increase the SNR by making $T_{1}$ smaller. In general the smaller $T_{1}$ would increase the bias of the estimate, and the frequency resolution would deteriorate. For white noise however the spectrum is flat, and no resolution is necessary.
In this section we used the particular case of white noise to conclude that the SNR of the PSD estimate is determined by the relative durations of the spatial and temporal integrations. We will now show that this general conclusion is also valid (approximately) for nonwhite noise processes.

\section{PSD Estimate Statistics: Nonwhite Noise}

In the case of nonwhite noise, it can be shown ${ }^{7}$ that the variance of $S(f)$ (the PSD estimate without any temporal integration) is approximately constant and thus independent of the spatial integration window $T_{1}$. In other words $S(f)$ is an inconsistent estimate as is the case for white noise. In the previous section we showed that for white noise the estimate becomes consistent if time integration is used in the detector, since the variance decreases monotonically as the ratio $T_{2} / T_{1}$ increases. To generalize our results for colored noise processes we will assume that the correlation function $R_{s}(\tau)$ of the process has negligible energy outside an interval $\Delta \tau$, centered at $\tau=0$. With this assumption the variation of the spectrum $W(f)$ within one frequency resolution element, $\Delta f \simeq 1 / T_{1}$, is bounded by

$$
\left|\frac{\Delta W(f)}{\Delta f}\right|<W_{\max } \Delta \tau=>|\Delta W(f)|<W_{\max } \frac{\Delta \tau}{T_{1}},
$$

according to the Bernstein theorem. ${ }^{7}$ If $\Delta \tau$ is small compared with $T_{1}$, we can neglect the variations of $W(f)$ within each frequency resolution element.

The general expression for the variance of $P(f)$ [Eq. (12)] can be rewritten as follows:

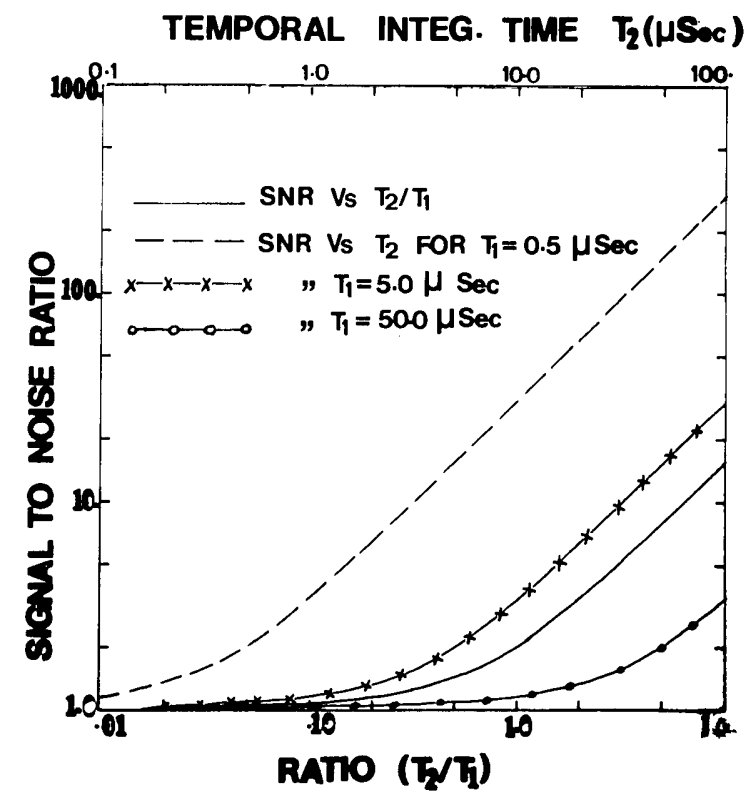

Fig. 2. SNR of the PSD estimate as a function of the ratio $\left(T_{2} / T_{1}\right)$ and as a function of time $T_{2}$ for $T_{1}=0.5,5.0$, and $50.0 \mu \mathrm{sec}$. 


$$
\begin{aligned}
\operatorname{Var}\{P(f)\}= & \frac{1}{T_{2}} \int_{-T_{2}}^{T_{2}}\left(1-\frac{|\gamma|}{T_{2}}\right) \mid \int_{-\infty}^{\infty} W\left(f-f^{\prime}\right) \exp \left[j 2 \pi \gamma\left(f-f^{\prime}\right)\right] \\
& \times\left. T_{1} \operatorname{sinc}^{2}\left(f^{\prime} T_{1}\right) d f^{\prime}\right|^{2} d \gamma+\frac{1}{T_{2}} \int_{-T_{2}}^{T_{2}}\left(1-\frac{|\gamma|}{T_{2}}\right) \mid \int_{-T_{1}}^{T_{1}} \\
& \times\left. R_{s}(\alpha+\gamma) \operatorname{sinc}\left[2 f\left(T_{1}-|\alpha|\right)\right]\left(1-\frac{|\alpha|}{T_{1}}\right) d \alpha\right|^{2} d \gamma
\end{aligned}
$$

The second term in the above equation can be neglected because the integral over $\alpha$ contains the product of a sinc function centered at $\alpha=T_{1}$ and a triangular function that reduces to zero as $\alpha=T_{1}$. Thus the value of this product is very small and, for $f>1 / T_{1}$, is a contribution that can be neglected. Equation (20) can be further simplified as follows.

$$
\begin{aligned}
\operatorname{Var}\{P(f)\} \simeq & \frac{1}{T_{2}} \int_{-T_{2}}^{T_{2}}\left(1-\frac{|\gamma|}{T_{2}}\right) \mid \int_{-\infty}^{\infty} W\left(f-f^{\prime}\right) \exp \left[j 2 \pi \gamma\left(f-f^{\prime}\right)\right. \\
& \times\left. T_{1} \operatorname{sinc}^{2}\left(f^{\prime} T_{1}\right) d f^{\prime}\right|^{2} d \gamma \\
& \simeq \frac{1}{T_{2}} \int_{-T_{2}}^{T_{2}}\left(1-\frac{|\gamma|}{T_{2}}\right) \mid W(f) \exp (j 2 \pi \gamma f) \\
& \times\left.\int_{-\infty}^{\infty} T_{1} \operatorname{sinc}^{2}\left(f^{\prime} T_{1}\right) \exp \left(-j 2 \pi \gamma f^{\prime}\right) d f^{\prime}\right|^{2} d \gamma .
\end{aligned}
$$

The last approximate expression was obtained by making use of the assumption that $W(f)$ has no appreciable variation over $\Delta f=1 / T_{1}$, which is also equal to the main lobe width of the $\operatorname{sinc}\left(f^{\prime} T_{1}\right)$ function in Eq. (21). Using the results derived in the Appendix, we find the variance of $P(f)$ to be

$$
\operatorname{Var}\{P(f)\} \simeq\left\{\begin{array}{c}
|W(f)|^{2}\left[1-(2 / 3)\left(T_{2} / T_{1}\right)+(1 / 6)\left(T_{2} / T_{1}\right)^{2}\right] \\
\text { for } T_{2}<T_{1}, \\
|W(f)|^{2}\left[(2 / 3)\left(T_{1} / T_{2}\right)-(1 / 6)\left(T_{1} / T_{2}\right)^{2}\right] \\
\text { for } T_{1}<T_{2} .
\end{array}\right.
$$

From Eq. (22) we see that for colored noise the variance exhibits the same dependance on the ratio $T_{1} / T_{2}$ as for white noise. The variance is also weighted at each frequency $f$ by $|W(f)|^{2}$ in this case.

The only assumption necessary to derive Eq. (22) is that the noise process must decorrelate in an interval $\Delta \tau$, which is small compared with the aperture $T_{1}$ of the AO cell. To complete our discussion we will examine the case where $\Delta \tau$ is comparable with or even larger than $T_{1}$. Since the bandwidth of such a random process would be $1 / \Delta \tau$, we would have a situation where the bandwidth of the signal is equal to or less than one resolution element of the spectrum analyzer. This represents a gross mismatch between the signal and the processor, and a buffer stage would be appropriate to compress the signal. The compressed signal would then have a small $\Delta \tau$ compared with $T_{1}$, and our previous results would be valid. However, if we wish to estimate the PSD of a wideband interference, compression is not practical, and we must know the variance of the PSD estimate of the narrowband signal. We model the spectrum of the narrowband process as a rectangular function centered at $f=f_{0}$ with width $\Delta f$. According to the above discussion, $\Delta f \simeq 1 / \Delta \tau \leq 1 / T_{1}$. We substitute the narrowband spectrum in the general ex- pression for the variance of $P(f)$ [Eq. (20)], and neglecting the contribution due to the second term as before we obtain

$$
\begin{aligned}
\operatorname{Var}\{P(f)\}= & \frac{T_{1}^{2}}{T_{2}} \int_{T_{2}}^{T_{2}}\left(1-\frac{|\gamma|}{T_{2}}\right) \mid \int_{f_{0}-\frac{\Delta f}{2}}^{f_{0}+\frac{\Delta f}{2}} \operatorname{sinc}^{2}\left[T_{1}\left(f-f^{\prime}\right)\right] \\
& \times\left.\exp \left(j 2 \pi \gamma f^{\prime}\right) d f^{\prime}\right|^{2} d \gamma .
\end{aligned}
$$

Evaluation of the above definite integral proves we can approximate the variance within a resolution element around $f=f_{0}$ by

$$
\begin{aligned}
\operatorname{Var}\{P\}= & 1+\operatorname{sinc}^{4}\left(\frac{\Delta f T_{1}}{2}\right)+2 \operatorname{sinc}^{2}\left(\frac{\Delta f T_{1}}{2}\right) \\
& \times\left\{\frac{2\left[1-\cos \left(\pi T_{2} \Delta f / 2\right)\right]}{\left(\pi T_{2} \Delta f / 2\right)^{2}}\right\} .
\end{aligned}
$$

The variance of the PSD estimate decreases for frequencies $f$ away from $f_{0}$. As can be evidenced from Eq. (24) this is an inconsistent estimate. Increasing $T_{2}$ does reduce the variance, however, in the limit as $T_{2} \rightarrow$ $\infty$, the variance has a finite value equal to

$$
\lim _{T_{2} \rightarrow \infty}[\operatorname{Var}\{P\}]=1+\operatorname{sinc}^{4}\left(\frac{\Delta f T_{1}}{2}\right) .
$$

The temporal integration in the narrowband case will result in an improvement of the SNR by a factor of 2, but it cannot provide arbitrarily high SNR as in the broadband case.

\section{Discussion}

The preceding analysis led us to the very important conclusion that only the combination of coherent (spatial) and incoherent (temporal) integration provides a low variance PSD estimate. Since the variance decreases as the ratio of $T_{2} / T_{1}$ increases, we must integrate in time for a period $T_{2}$ much higher than $T_{1}$. On the other hand the frequency resolution of the estimate is $\sim 1 / T_{1}$ according to Eq. (9). To obtain an estimate that has both fine frequency resolution (low bias) and small variance we need a very long incoherent integration. Thus there is a practical trade-off between frequency resolution and variance in the usual case where we have a limited time in which to produce the PSD estimate.

Our presentation has been descriptive, and we assumed an ideal AO system, but we believe our results will be very useful in the design of $\mathrm{AO}$ spectrum analyzers. For instance, let us assume that there is additive signal independent noise $\{n\}$ with zero mean that is introduced at the detector of the AO system. The SNR of the estimate in this case is

$$
\mathrm{SNR}=\frac{|E\{P(f)\}|^{2}}{\operatorname{Var}\{P(f)\}+\operatorname{Var}\{n\}} .
$$

It is imperative to determine the variance of $P(f)$ because, if an inappropriate combination of $T_{2}$ and $T_{1}$ is chosen, its contribution to the total SNR will completely dominate the statistics of the estimate, and hence only marginal improvement will result by taking additional steps to minimize the detector noise.

The analysis in this paper can be easily extended to 
describe more complicated scenarios than the one described here. Let us assume that we wish to estimate the PSD of a random process $\left\{s_{1}\right\}$ in the presence of additive, signal-independent broadband noise $\left\{s_{2}\right\}$ with known PSD. Then the SNR of the estimate is

$$
\begin{aligned}
\mathrm{SNR} & =\frac{\left|E\left\{P_{1}(f)\right\}\right|^{2}}{\operatorname{Var}\left\{P_{1}(f)\right\}+\operatorname{Var}\left\{P_{2}(f)\right\}} \\
& \simeq \begin{cases}{\left[\frac{\left|W_{1}(f)\right|^{2}}{\left|W_{1}(f)\right|^{2}+\left|W_{2}(f)\right|^{2}}\right]\left[1-2 / 3\left(T_{2} / T_{1}+1 / 6\left(\mathrm{~T}_{2} / \mathrm{T}_{1}\right)^{2}\right]^{-1}\right.} & \text { for } T_{2}<T_{1} \\
{\left[\frac{\left|W_{1}(f)\right|^{2}}{\left|W_{1}(f)\right|^{2}+\left|W_{2}(f)\right|^{2}}\right]\left[2 / 3\left(T_{1} / T_{2}\right)-1 / 6\left(T_{1} / T_{2}\right)^{2}\right]^{-1}} & \text { for } T_{1}<T_{2} .\end{cases}
\end{aligned}
$$

In this case the additive noise has deteriorated the SNR for a fixed ratio $T_{1} / T_{2}$, but the estimate is still consistent since the SNR tends to infinity as $T_{2}$ becomes much larger than $T_{1}$. Thus we can compensate the variance due to $\left\{s_{2}\right\}$ by making the ratio $T_{2} / T_{1}$ larger if $\left\{s_{2}\right\}$ is not narrowband.

Finally the combination of spatial and temporal integration afforded by AO systems gives us the opportunity to use any a priori information available about the signal or the interference to improve the output SNR. This can be done by placing a temporal window (filter) in the system. This is analogous in some respects to the spatial window commonly used to reduce the side-lobe level and increase the resolution.

The authors gratefully acknowledge the support of the National Science Foundation under contract ENG-78-20038.

\section{Appendix: Derivation of Eq. (16)}

To obtain the variance of $P(f)$ for a white noise process, we substitute $R_{s}(\tau)=R_{0} \delta(\tau)$ in Eq. (12):

$$
\begin{aligned}
\operatorname{Var}\{P(f)\}= & \frac{R_{0}^{2}}{\mathrm{~T}_{1}^{2} T_{2}^{2}} \iint_{-T_{2} / 2}^{+T_{2} / 2} \\
& \times d t d u\left[\iiint \int_{-T_{1} / 2}^{T_{1} / 2} \exp [-j 2 \pi f(x-y+p-q)]\right. \\
& \cdot\{\delta(p-x+u-t) \delta(q-y+u-t) \\
& +\delta(q-x+u-t) \delta(p-y+u-t)\} d x d y d p d q]
\end{aligned}
$$

With $u-t=\tau$ we obtain

$$
\begin{aligned}
\operatorname{Var}\{P(f)\}= & \frac{R_{0}^{2}}{T_{1}^{2} T_{2}} \int_{-T_{2}}^{T_{2}}\left(1-\frac{|\tau|}{T_{2}}\right) \\
& \times\left|T_{1} \int_{-T_{1}}^{T_{1}}\left(1-\frac{|\alpha|}{T_{1}}\right) \delta(\alpha+\tau) \exp (-j 2 \pi f \alpha) d \alpha\right|^{2} d \tau \\
& +\frac{R_{0}^{2}}{T_{1}^{2} T_{2}} \int_{-T_{2}}^{T_{2}}\left(1-\frac{|\tau|}{T_{2}}\right) \\
& \times \mid \iint_{-T_{1} / 2}^{T_{1} / 2} \delta(p-x+\tau) \\
& \times\left.\exp [-j 2 \pi f(p+x)] d p d x\right|^{2} d \tau,
\end{aligned}
$$

where $\alpha=q-x$ and $p-y$. With $u=p-q$, Eq. (A2) becomes

$$
\begin{aligned}
\operatorname{Var}\{P(f)\}= & \frac{1}{T_{2}} \int_{-T_{2}}^{T_{2}}\left(1-\frac{|\tau|}{T_{2}}\right) \\
& \times\left|\int_{-T_{1}}^{T_{1}}\left(1-\frac{|\alpha|}{T_{1}}\right) \delta(\alpha+\tau) \exp (-j 2 \pi f \alpha) d \alpha\right|^{2} d \tau \\
& +\frac{1}{4 T_{1}^{2} T_{2}} \int_{-T_{2}}^{T_{2}}\left(1-\frac{|\tau|}{T_{2}}\right) \\
& \times\left|\int_{-T_{1}}^{T_{1}} \frac{\sin 2 \pi f\left(T_{1}-|u|\right)}{\pi f} \delta(u+\tau) d u\right|^{2} d \tau .
\end{aligned}
$$

For $T_{1}>T_{2}$, Eq. (A3) can be evaluated as follows:

$$
\begin{aligned}
\operatorname{Var}\{P(f)\}= & \frac{1}{T_{2}} \int_{-T_{2}}^{T_{2}}\left(1-\frac{|\tau|}{T_{2}}\right)\left(1-\frac{|\tau|}{T_{1}}\right)^{2} d \tau \\
& +\frac{1}{T_{1}^{2} T_{2}} \int_{-T_{2}}^{T_{2}}\left(1-\frac{|\tau|}{T_{2}}\right)\left[\frac{\sin 2 \pi f\left(T_{1}-|\tau|\right)}{2 \pi f}\right]^{2} d \tau \\
= & {\left[1-(2 / 3)\left(\frac{T_{2}}{T_{1}}\right)+(1 / 6)\left(\frac{T_{2}}{T_{1}}\right)^{2}\right]+\frac{1}{4 \pi^{2} f^{2} T_{1}^{2} T_{2}^{2}} } \\
& \times\left\{\frac{T_{2}^{2}}{2}+\frac{T_{2}}{4 \pi f} \sin 4 \pi f T_{1}-\frac{T_{2}}{4 \pi f} \sin 4 \pi\left(T_{1}-T_{2}\right)\right. \\
& \left.+\frac{\cos 4 \pi f\left(T_{1}-T_{2}\right)}{(4 \pi f)^{2}}-\frac{\cos 4 \pi f T_{1}}{(4 \pi f)^{2}}\right\}
\end{aligned}
$$

The variance increases at lower frequencies, but for $f=1 / T_{1}$ the portion of the variance that is frequency dependent is only $1 / 40$ of the total variance, and at higher frequencies the contribution decreases quadratically. Thus for $f>1 / T_{1}$, Eq. (A4) for all practical purposes is given by

$$
\operatorname{Var}\{P(f)\} \simeq 1-(2 / 3)\left(\frac{T_{2}}{T_{1}}\right)+(1 / 6)\left(\frac{T_{2}}{T_{1}}\right)^{2} \quad \text { for } \quad T_{2}<T_{1} .
$$

For $T_{2}<T_{1}$, Eq. (A3) is evaluated in a similar fashion, and we obtain

$$
\operatorname{Var}\{P(f)\} \simeq(2 / 3)\left(\frac{T_{1}}{T_{2}}\right)-(1 / 6)\left(\frac{T_{1}}{T_{2}}\right)^{2} \text { for } T_{1}<T_{2},
$$

Eqs. (A5) and (A6) constitute Eq. (16).

\section{References}

1. G. M. Jenkins and D. G. Watts, Spectral Analysis and its Applications (Holden-Day, San Francisco, 1968).

2. L. H. Koopmans, The Spectral Analysis of Time Series (Academic, New York, 1974).

3. I. C. Chang, IEEE Trans. Sonics Ultrason. SU-23, 2 (1976).

4. D. L. Hecht, Opt. Eng. 16, 461 (1977).

5. D. B. Anderson, IEEE Spectrum 15 (12), 22 (1978).

6. A. Papoulis, Probability, Random Variable, and Stochastic Processes (McGraw-Hill, New York, 1965).

7. A. Papoulis, Systems and Transforms with Applications in Optics (McGraw-Hill, New York, 1968). 